\title{
Correlative Microscopy and Spectroscopy of Buried Interfaces in Tooth Enamel
}

\author{
Lyle M. Gordon, Michael J. Cohen and Derk Joester
}

Department of Materials Science and Engineering, Northwestern University, Evanston, IL 60208 USA

Tooth decay, also known as dental caries, is the most frequent infectious disease in humans. With billions of dollars being spent on dental services in the US alone, there is no question that a significant demand exists for improved dental treatments. Tooth enamel is composed of layers of woven $3-5 \mu \mathrm{m}$ diameter rods, each comprised of thousands of hydroxyapatite nanowires. However, despite decades of research on enamel, the complex nanoscale structure and chemistry of the tissue is still not fully understood, in particular the nature of the intercrystalline space. The disordered material at the grain boundaries has a significant influence on the final properties of the composite. A more complete understanding of the interphase structure and chemistry is a fundamental milestone for preventing and effectively treating dental caries.

Atom-probe tomography (APT) is uniquely capable of providing the necessary structural insight at the atomic scale by directly probing the location and chemical identity of the atoms within a small sample of material [1]. APT consists of a field-ion microscope coupled to a time-of-flight mass spectrometer, providing chemical and three-dimensional positional information of ions successively field evaporated from a sharp sample. The advent of ultraviolet laser pulsing has widely enlarged the scope of APT to include a range of insulating materials, including hybrid organic-inorganic materials [2]. We recently demonstrated the remarkable ability of APT to map the structure and chemistry of buried interfaces in biological minerals in the magnetite cusp of the chiton tooth [3] and vertebrate bone and dentin [4].

We will report here on the application of APT in combination with transmission electron microscopy (TEM) and X-ray absorption spectroscopy (XAS) to characterize rodent incisor enamel. APT samples were prepared with standard FIB lift out and sharpening protocols [5] from polished cross sections of mouse incisor enamel. APT reconstructions clearly reveal the individual 10-20 nm diameter faceted crystallites. The unique sensitivity of APT revealed numerous inorganic substituents (such as $\mathrm{Na}^{+}, \mathrm{Mg}^{2+}$, $\mathrm{CO}_{3}{ }^{2-}$ ) and characteristic nitrogen-containing fragments of organic macromolecules (Figure 1B-C). Correlative TEM imaging of select samples was performed on an aberration corrected TEM at Argonne National Laboratory. Grain boundaries observed in TEM were consistent with segregation observed in the APT reconstructions. (Figure 1D-E). Following the deposition of the apatetic enamel mineral, an iron-rich phase is deposited on the surface of the enamel in certain rodents. Using APT we observed that the iron-rich deposits are localized primarily at the apatite grain boundaries near the surface.

While APT provides a detailed picture of the distribution of elements with sub-nanometer resolution, information regarding the local chemistry including bond distance, coordination number and valence state of each element is not available. Synchrotron XAS of iron-rich enamel surface provides a sensitive element-specific tool to probe the local chemical environment. We will discuss combining structural insight from APT reconstructions with chemical information gleaned from XAS and our preliminary model of iron-oxide-hardened rodent tooth enamel. 
[1] T.F. Kelly, D.J. Larson, Ann. Rev. Mat. Res. 2012, 42: 1-31

[2] D. Joester, et al. Microscopy Today. 2012, 20: (2), 26-31

[3] L.M. Gordon, D. Joester, Nature 2011, 469, 194-197

[4] L.M. Gordon, L. Tran, D. Joester, ACS Nano, 2012, 6 (12) 10667-10675

[5] K. Thompson et al. Ultramicroscopy, 2007, 107 (2-3) 131-139

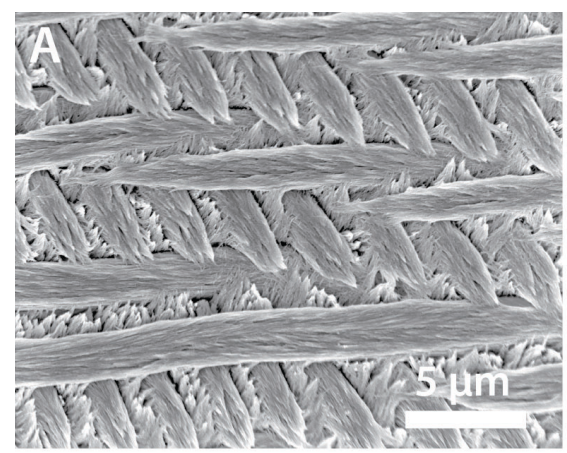

C
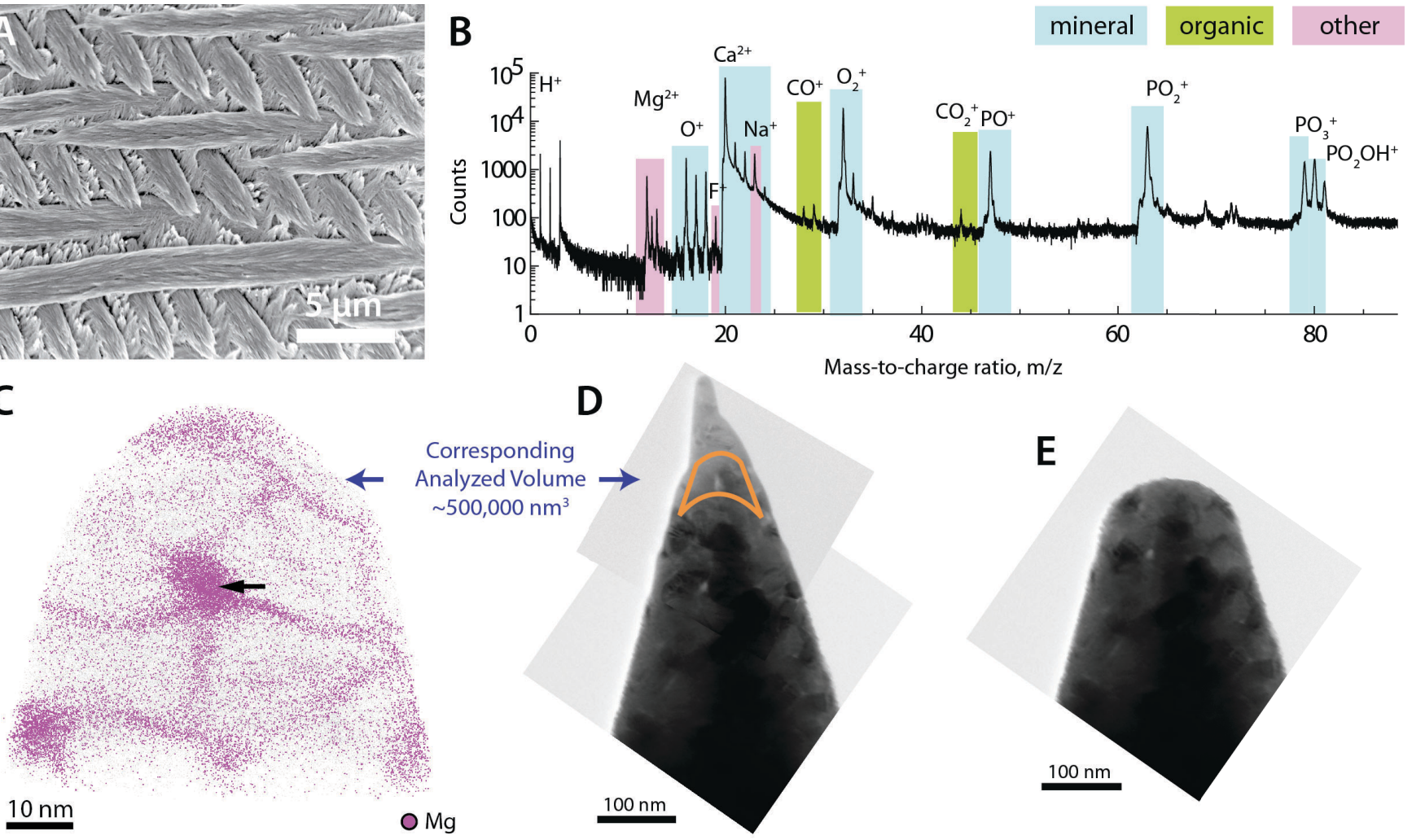

Figure 1. A. Scanning electron micrograph of acid etched mouse enamel cross section showing overlapping rods composed of thousands of individual oriented hydroxyapatite nanocrystals. B. APT mass spectra of mouse enamel sample highlighting apatite-derived calcium and phosphate ions and inorganic chemical substituents including $\mathrm{Mg}^{2+}, \mathrm{Na}^{+}, \mathrm{F}^{+}$and $\mathrm{CO}_{\mathrm{x}}^{+}$. C. 3D reconstruction of magnesium ion positions showing localization at the grain boundaries. D/E. TEM image of corresponding mouse enamel tip before (D) and after (E) atom probe analysis, corresponding grain boundaries and multiple crystal junctions are visible. 